\title{
Impact of Brief Exposure to Drugs with Antifungal Properties on the Susceptibility of Oral Candida dubliniensis Isolates to Lysozyme and Lactoferrin
}

\author{
Arjuna Nishantha B. Ellepola ${ }^{a}$ Ranil Samantha Dassanayake ${ }^{b}$ Ziauddin Khan $^{c}$ \\ ${ }^{a}$ Faculty of Dentistry, Health Sciences Center, Kuwait University, Safat, Kuwait; ${ }^{b}$ Faculty of Science, University of \\ Colombo, Colombo, Sri Lanka; ' ${ }^{\mathrm{F}}$ aculty of Medicine, Health Sciences Center, Kuwait University, Safat, Kuwait
}

\section{Significance of the Study}

- Lysozyme and lactoferrin are salivary constituents with anti-candidal activity. Candida infections are managed with antimycotic drugs; there is no information on the impact of these drugs (i.e., synergism or antagonism) on the susceptibility of Candida dubliniensis to lysozyme and lactoferrin. This study reveals that exposure to antifungal drugs increases the susceptibility of $C$. dubliniensis to lysozyme and lactoferrin.

\section{Keywords}

Antifungal agents · Candida dubliniensis · Lactoferrin · Lysozyme

\footnotetext{
Abstract

Objective: Lysozyme and lactoferrin have anti-candidal activity. Candida dubliniensis is associated with oral candidiasis. Candida infections are managed with nystatin, amphotericin $B$, caspofungin, ketoconazole, fluconazole, and chlorhexidine. Candida species undergo a brief exposure to therapeutic agents in the mouth. There is no data on the influence of limited exposure to antimycotics on the sensitivity of C. dubliniensis to lactoferrin and lysozyme. Hence, this study observed the changes in the sensitivity of $C$. dubliniensis to anticandidal action of lactoferrin and lysozyme after transitory exposure to sub-lethal concentrations of antifungals. Materials and Methods: After determination of the minimum inhibitory concentration (MIC), 20 C. dubliniensis isolates were
}

exposed to twice the concentration of MIC of nystatin, amphotericin B, caspofungin, ketoconazole, fluconazole, and chlorhexidine for $1 \mathrm{~h}$. Drugs were removed by dilution and thereafter the susceptibility of these isolates to lysozyme and lactoferrin was determined by colony-forming unit quantification assay. Results: Exposure of $C$. dubliniensis to nystatin, amphotericin B, caspofungin, ketoconazole, fluconazole, and chlorhexidine resulted in an increase in susceptibility to lysozyme by $9.45,30.82,30.04,50.64,55.60$, and $50.18 \%$, respectively ( $p<0.05$ to $p<0.001$ ). Exposure of C. dubliniensis to nystatin, amphotericin B, caspofungin, ketoconazole, fluconazole, and chlorhexidine resulted in an increase in susceptibility to lactoferrin by $13.54,16.43,17.58$, $19.60,21.32$, and 18.73, respectively ( $p<0.05$ to $p<0.001$ ). Conclusion: Brief exposure to nystatin, amphotericin $B$, caspofungin, ketoconazole, fluconazole, and chlorhexidine enhances the antifungal effect of lysozyme and lactoferrin on C. dubliniensis isolates in vitro.

(c) 2018 The Author(s) Published by S. Karger AG, Basel

\begin{tabular}{|c|c|c|}
\hline KARGER & $\begin{array}{l}\text { (0) } 2018 \text { The Author(s) } \\
\text { Published by S. Karger AG, Basel }\end{array}$ & $\begin{array}{l}\text { Karger } \\
\text { Open access }\end{array}$ \\
\hline $\begin{array}{l}\text { E-Mail karger@karger.com } \\
\text { www.karger.com/mpp }\end{array}$ & $\begin{array}{l}\text { This is an Open Access article licensed } \\
\text { Attribution-NonCommercial-4.0 Inter } \\
\text { (http://www.karger.com/Services/Ope } \\
\text { the online version of the article only. } \mathrm{U} \\
\text { mercial purposes requires written perr }\end{array}$ & $\begin{array}{l}\text { r the Creative Commons } \\
\text { nal License (CC BY-NC) } \\
\text { essLicense), applicable to } \\
\text { and distribution for com- } \\
\text { on. }\end{array}$ \\
\hline
\end{tabular}




\section{Introduction}

Candida dubliniensis is currently widely acknowledged as an opportunistic pathogen allied with recurrent oral candidiasis in patients with acquired immunodeficiency syndrome (AIDS). It has also been isolated from the niches of the oral milieu of diabetic and cystic fibrosis patients. C. dubliniensis has been isolated from upper respiratory tract specimens and blood as well as from a case of endocarditis involving a prosthetic aortic valve, which indicates that it can disseminate to other sites [1-4]. Moreover, cases of spondylodiscitis, multifocal osteomyelitis, and leptomeningeal disease have been shown to be caused by C. dubliniensis [5-7]. Additionally, resistance to fluconazole has been observed in C. dubliniensis isolates obtained from AIDS patients and stable resistance to this triazole can be readily produced in C. dubliniensis following exposure to this drug in vitro [8]. Likewise, a breakthrough in C. dubliniensis fungemia was made in a patient during sustained exposure to voriconazole [9]. Longitudinal genotyping of $C$. dubliniensis isolates from patients with HIV infection has shown resistance to itraconazole, even in the absence of prior azole therapy [10]. C. dubliniensis strains resistant to 5-fluorocytosine have also been isolated in Kuwait and in the Middle East region $[11,12]$.

Human whole saliva is accepted as a principal source of numerous antimicrobial substances, such as lactoferrin and lysozyme. These nonimmune protective determinants are well known for their functionality in regulating the quantity and quality of microflora on mucosal surfaces [13]. Lactoferrin, an iron-binding glycoprotein, is found in granules of polymorphonuclear leukocytes and in saliva [13]. Lysozyme, which is present in the oral environment, arises from the salivary glands, gingival tissue, and crevicular fluid [13]. In vitro investigations have validated bacteriostatic, bactericidal, and anticandidal activity of apo-lactoferrin, which is an iron-free lactoferrin $[14,15]$. Lysozyme has also been shown to exert antimicrobial activity on a range of oral microorganisms in vitro including Candida species [15].

A plethora of drugs with antimycotic pharmacodynamics are available for the treatment of oral candidiasis. These include the polyene antifungals such as nystatin and amphotericin $\mathrm{B}$, echinocandins such as caspofungin, the azoles such as ketoconazole (an imidazole) and fluconazole (a triazole), and the widely used oral mouth wash chlorhexidine gluconate $[16,17]$. However, the diluting impact of saliva and the cleansing outcome of the oral musculature in the oral environment tend to reduce the availability of these pharmaceuticals to levels below their effective therapeutic concentrations, thus reducing their therapeutic efficacy $[16,17]$. Hence, the opportunistic Candida may undergo a brief exposure to antifungal pharmaceuticals during therapy, a scenario all too familiar in the niches of the oral cavity $[16,17]$.

Recent research has demonstrated that brief exposure to sub-therapeutic concentrations of drugs with antimycotic pharmacodynamics such as nystatin, amphotericin $\mathrm{B}$, caspofungin, ketoconazole, fluconazole, and chlorhexidine gluconate could modulate the pathogenic features of oral Candida, ranging from adhesion to denture acrylic and buccal epithelial cells, cell surface hydrophobicity, germ tube formation, and production of pathogenic enzymes such as candidal hemolysin and phospholipase [18-23]. However, only one study has investigated the impact of lysozyme and lactoferrin on oral Candida (i.e., Candida albicans and Candida tropicalis) pre-exposed to three antimycotic agents such as nystatin, amphotericin B, and fluconazole, for a brief period [24]. Such an impact on the clinically important $C$. dubliniensis isolates, with a wide array of different drugs with antifungal properties, has not been investigated so far. Hence, based on these considerations, supported by the findings of a recent prevalence study which showed that oral C. dubliniensis isolates had the highest prevalence among non-albicans oral Candida species in Kuwait [25], the main aim of this study was to investigate the changes in susceptibility to lactoferrin and lysozyme of 20 oral isolates of C. dubliniensis after brief exposure ( $1 \mathrm{~h}$ ) to sub-therapeutic concentrations of nystatin, amphotericin $\mathrm{B}$, caspofungin, ketoconazole, fluconazole, and chlorhexidine gluconate. In addition, as $C$. dubliniensis and C. albicans are phenotypically similar Candida species, C. albicans oral isolates obtained from Kuwait were also studied for the purpose of comparison of results between these two Candida species.

\section{Materials and Methods}

\section{Organisms}

Twenty oral isolates each of C. dubliniensis and C. albicans maintained as stock cultures and stored in the Oral Microbiology Laboratory, Faculty of Dentistry and in the Mycology Unit, Faculty of Medicine, Kuwait University were used. The stock cultures of Candida were from the oral rinse samples obtained in a previous investigation, from patients attending the Kuwait University Dental Clinic for dental treatment [25]. These patients were healthy adults and none of the patients from whom the isolates were obtained had oral candidiasis. Identification and confirmation of the isolates were carried out as per our previous investigations [18- 
23]. Initially, all Candida isolates were tested for germ tube formation. Their colony characteristics were also determined by means of CHROMagar Candida medium (Becton Dickinson and Company, Sparks, MD, USA) and carbohydrate assimilation profiles were obtained using the VITEK 2 Candida identification system (BioMérieux, France). Subsequently, identification of these isolates was confirmed further by using semi-nested PCR amplification of the internal transcribed spacer 2 region of $\mathrm{rDNA}$ followed by direct DNA sequencing of the internal transcribed spacer region of rDNA. The semi-nested PCR amplification was performed as described previously [18-23]. Briefly, amplification with universal fungal primers was carried out in $50 \mu \mathrm{L}$ containing $1 \times \mathrm{Am}$ pliTaq PCR buffer I, 1 U AmpliTaq DNA polymerase, deionized water, $10 \mathrm{pmol}$ each of CTSF and CTSR primers, $1 \mu \mathrm{L}$ of DNA and $0.1 \mathrm{mM}$ of each dNTP. After amplification in the first step, $1 \mu \mathrm{L}$ of the product was further amplified using CTSR and C. dubliniensisspecific CDDET primer yielding an amplicon of $105 \mathrm{bp}$ as described previously [18-23]. Thereafter, the amplified products (20 $\mu \mathrm{L}$ ) were resolved by electrophoresis in agarose gels.

\section{Antifungal Agents and Media}

Antifungal agents were prepared as done previously [18-23]. Briefly, nystatin, amphotericin B, ketoconazole, and fluconazole (Sigma, St. Louis, MO, USA) were dissolved in dimethylsulfoxide (DMSO). Caspofungin (Merck and Company Inc., Waterhouse Station, NJ, USA) was dissolved in sterile distilled water. These antimycotic agents were prepared initially as stocks of 10,000 $\mu \mathrm{g} /$ $\mathrm{mL}$ and stored at $-20^{\circ} \mathrm{C}$ before use. They were suspended/diluted in the following medium during the exposure period $(1 \mathrm{~h})$. RPMI1640 medium buffered with 0.165 M MOPS (3-( $N$-morpholino) propanesulfonic acid) containing L-glutamine and lacking sodium bicarbonate (Sigma, USA) was dissolved in $1 \mathrm{~L}$ of sterile distilled water and adjusted to a $\mathrm{pH}$ of 7.2 and filter-sterilized. This medium was stored at $2-8{ }^{\circ} \mathrm{C}$ for $2-3$ months. The stock solution was used to obtain drug concentrations (i.e., $2 \times$ minimum inhibitory concentration $[\mathrm{MIC}]$ ). Chlorhexidine gluconate $0.2 \%$ (GlaxoSmithKline, Brentford, UK) was dissolved in sterile phosphate-buffered saline (PBS) at pH 7.2 and was diluted to obtain a concentration of $0.005 \%$ prior to each experiment as previously described [18].

\section{Determination of MIC}

MIC values for nystatin and chlorhexidine were obtained by the broth dilution technique by performing twofold serial dilutions of the drugs in microtiter plates using an inoculum of $1-5 \times 10^{5} \mathrm{col}-$ ony-forming units (CFU)/mL [18, 22]. The MIC values were obtained visually and spectrophotometrically at $595 \mathrm{~nm}$ following $24 \mathrm{~h}$ of incubation at $37^{\circ} \mathrm{C}$. The MIC was defined as the lowest concentration of the drug that inhibited growth of Candida, as indicated by the absence of turbidity (optically clear). C. albicans ATCC 90028 and Candida parapsilosis ATCC 22019 were used as reference strains.

The MIC values for amphotericin B, caspofungin, ketoconazole, and fluconazole were determined by E tests as described previously according to the manufacturer's recommendations ( $\mathrm{AB}$ BIODISK, Solna, Sweden) [18-23]. Each test isolate was freshly subcultured. Five isolated colonies were uniformly suspended in sterile saline, and turbidity was adjusted to $0.5 \mathrm{McF}$ arland standard $\left(1-5 \times 10^{6}\right.$ cells $\left./ \mathrm{mL}\right)$. This inoculum was swabbed onto the agar plates of $150 \mathrm{~mm}$ diameter and allowed to dry for 10-15 min. Thereafter, the E test strips were placed. RPMI-1640 agar supple- mented with $2 \%$ glucose and buffered with MOPS (0.165 M; pH 7.0) was used for susceptibility testing according to the method recommended by the Clinical and Laboratory Standards Institute (formerly National Committee for Clinical Laboratory Standards; M27-A2). The plates were incubated at $35^{\circ} \mathrm{C}$, and MIC was read after $24-48 \mathrm{~h}$ of incubation. The point where inhibition ellipses intercepted the scale on the antifungal strip was taken as the MIC for each test isolate. Reference strains of C. albicans, ATCC 90028, and C. parapsilosis, ATCC 22019, were used for quality control of the susceptibility testing. Interpretive susceptibility breakpoints for fluconazole were those recommended by Clinical and Laboratory Standards Institute document M27-A2. Due to the lack of defined susceptibility breakpoints for amphotericin B and ketoconazole, an isolate was considered susceptible with an MIC breakpoint of $\leq 1.0 \mu \mathrm{g} / \mathrm{mL}$ for amphotericin B and $0.125 \mu \mathrm{g} / \mathrm{mL}$ for ketoconazole as determined in previous studies [18-22, 25]. Isolates with MIC of $\leq 2.0 \mu \mathrm{g} / \mathrm{mL}$ for caspofungin were considered as susceptible $[23,25]$.

\section{Preparation of Yeast Cell Suspension and Exposure to}

Antimycotic Agents

A previously described method was used for this purpose [1823]. Briefly, yeast cells maintained on Sabouraud's dextrose agar were inoculated onto fresh plates and incubated overnight at $37^{\circ} \mathrm{C}$ for $24 \mathrm{~h}$ prior to use. The organisms were harvested and a cell suspension prepared in sterile PBS of $\mathrm{pH} 7.4$ to an optical density of 1.5 at $520 \mathrm{~nm}$. From this cell suspension, $1 \mathrm{~mL}$ was added to tubes containing $4 \mathrm{~mL}$ of RPMI broth (control) and $4 \mathrm{~mL}$ of RPMI/drug solution (test), in which the drug concentrations were twice the MIC values. This yielded a cell suspension of $10^{6}$ cells $\mathrm{mL}^{-1}$ in each assay tube. The tubes were then incubated at $37^{\circ} \mathrm{C}$ for $1 \mathrm{~h}$ in a rotary incubator. Following this limited exposure, the drugs were removed by two cycles of dilution with sterile PBS and centrifugation for $10 \mathrm{~min}$ at 3,000 $\mathrm{g}$. Afterwards the supernatant was completely decanted, and the pellets were resuspended in $5 \mathrm{~mL}$ of sterile PBS. This procedure for drug removal has shown to reduce the concentration of the drug as much as 10,000-fold, thereby minimizing any carry-over effect of the drug [18-23]. Viable counts of the control and the test were performed after each procedure of drug removal. As the procedure of drug removal effectively eliminated any carry-over effect, there was virtually no difference in the viable counts of the control and the tests following exposure to already diluted sub-therapeutic concentrations of the drug as observed in previous studies [18-23].

The Candida pellets obtained were washed twice by suspending in ice-cold $0.05 \mathrm{~mm}$ potassium chloride $(\mathrm{KCl})$, buffered to $\mathrm{pH} 7.0$ with potassium hydroxide $(\mathrm{KOH})$ and thereafter harvested by centrifugation at $3,500 \mathrm{~g}$ for $5 \mathrm{~min}$. The yeasts were resuspended in the buffered $\mathrm{KCl}$ to give a final concentration $5 \times 10^{6}$ cells $\mathrm{mL}^{-1}$ [24].

\section{Lactoferrin and Lysozyme}

Human apo-lactoferrin (Sigma Chemical Co., Poole, UK) was used for the study. A stock solution of apo-lactoferrin (0.016 $\mathrm{g}$ of the protein in $2 \mathrm{~mL}$ sterile distilled water) was prepared at a dilution of $200 \mu \mathrm{g} \mathrm{mL} \mathrm{mL}^{-1}$ with sterile distilled water as previously performed [24]. Hen egg-white lysozyme (Sigma Chemical Co.) was similarly used for all the experiments. A stock solution of lysozyme ( $0.016 \mathrm{~g}$ of the protein in $2 \mathrm{~mL}$ sterile distilled water) was prepared at a dilution of $300 \mu \mathrm{g} \mathrm{mL}^{-1}$ as done previously [24]. 
Lactoferrin- and Lysozyme-Induced Antifungal Assays

The antifungal effect of either lactoferrin $\left(\mathrm{F}_{\mathrm{LF}}\right)$ or lysozyme $\left(\mathrm{F}_{\mathrm{LZ}}\right)$ on the isolates was determined with minor modifications [24, 26]. Briefly, suspensions of either $200 \mu \mathrm{g} \mathrm{mL}^{-1}$ of apo-lactoferrin or $100 \mu \mathrm{g} \mathrm{mL}^{-1}$ of lysozyme, and $100 \mu \mathrm{L}$ of the Candida suspension were dispensed into sterile tubes containing $800 \mu \mathrm{L}$ of $0.05 \mathrm{mM}$ phosphate-buffered $\mathrm{KCl}(0.05 \mathrm{mM}$; $\mathrm{pH} 7.0)$ to yield a cell concentration of $5 \times 10^{5} \mathrm{~mL}^{-1}$ yeasts. Thus, the final concentrations of apo-lactoferrin and lysozyme in the suspensions were 20 and $30 \mu \mathrm{g}$ $\mathrm{mL}^{-1}$, respectively. In the control sample, $100 \mu \mathrm{L}$ sterile distilled water was substituted for the protein. Both the lactoferrin- or lysozyme-containing tubes and the control tubes were then incubated at $37^{\circ} \mathrm{C}$ for $1 \mathrm{~h}$ with gentle shaking. After incubation, the tubes were carefully vortexed; $100-\mu \mathrm{L}$ samples were diluted 1:50 and plated on Sabouraud's dextrose agar and the resultant CFU were quantified after $48 \mathrm{~h}$ of incubation at $37^{\circ} \mathrm{C}$.

The antifungal activity of lactoferrin $\left(\mathrm{F}_{\mathrm{LF}}\right)$ or lysozyme $\left(\mathrm{F}_{\mathrm{LZ}}\right)$ was computed using the formula for both drug-exposed (control [i.e., without lactoferrin or lysozyme] and with lactoferrin or lysozyme) and unexposed isolates (control [i.e., without lactoferrin or lysozyme] and with lactoferrin or lysozyme):

$\mathrm{F}_{\mathrm{LF}}$ or $\mathrm{F}_{\mathrm{LZ}}=\left(\mathrm{CFU} \mathrm{mL}^{-1}\right.$ of control suspension $-\mathrm{CFU} \mathrm{mL} \mathrm{m}^{-1}$ of lactoferrin or lysozyme suspension)/CFU $\mathrm{mL}^{-1}$ of control suspension.

Thus, the higher the $\mathrm{F}_{\mathrm{LF}}$ or $\mathrm{F}_{\mathrm{LZ}}$ value, the higher the sensitivity of the isolates to either protein.

All experiments were repeated on three separate occasions with duplicate determinations on each occasion. C. dubliniensis reference strain CD36 and C. albicans reference strain ATCC 90028 were also used in the above assays.

Statistical Analysis

The data obtained from all three assays in duplicate were analyzed as done previously [18-23] using the ANOVA Dunnett $t$ test, which treats one group as a control (unexposed to drugs) and compares all other groups (exposed to drugs) against it. A $p$ value of $<0.05$ was considered statistically significant.

\section{Results}

The MIC $(\mu \mathrm{g} / \mathrm{mL})$ values of 20 isolates of C. dubliniensis and C. albicans to nystatin, amphotericin B, caspofungin, ketoconazole, fluconazole, and chlorhexidine gluconate were: nystatin: 0.09-0.78; amphotericin B: $0.002-0.125$; caspofungin: $0.003-0.19$; ketoconazole: 0.002-0.012; fluconazole: $0.016-0.38$; and chlorhexidine gluconate: $0.00125 \%$. These values were within the normal MIC range for the Candida species for each of the drugs tested as seen in previous studies as well [18-23]. Thus, none of the isolates was resistant to the tested drugs.

The mean $\mathrm{F}_{\mathrm{LZ}}$ value of $C$. dubliniensis isolates unexposed to antimycotics was 0.550 . Brief exposure to nystatin, amphotericin B, caspofungin, ketoconazole, fluconazole, and chlorhexidine gluconate significantly $(p<$
0.05 to $p<0.001)$ increased the susceptibility of the isolates to lysozyme, yielding mean $\mathrm{F}_{\mathrm{LZ}}$ values of 0.602 , $0.714,0.710,0.821,0.848$, and 0.820 , respectively (Table 1). Thus, exposure to nystatin, amphotericin $B$, caspofungin, ketoconazole, fluconazole, and chlorhexidine gluconate provided mean percentage increase in susceptibility to lysozyme by $9.45,30.82,30.04,50.64,55.60$, and $50.18 \%$, respectively (Table 1 ). The mean $\mathrm{F}_{\mathrm{LZ}}$ value of $C$. albicans isolates unexposed to antimycotics was 0.562 . Brief exposure to nystatin, amphotericin B, caspofungin, ketoconazole, fluconazole, and chlorhexidine gluconate significantly ( $p<0.05$ to $p<0.001)$ increased the susceptibility of the isolates to lysozyme, yielding mean $\mathrm{F}_{\mathrm{LZ}}$ values of $0.612,0.712,0.711,0.821,0.853$, and 0.817 , respectively (Table 1). Thus, exposure to nystatin, amphotericin $\mathrm{B}$, caspofungin, ketoconazole, fluconazole, and chlorhexidine gluconate provided mean percentage increase of susceptibility to lysozyme by $8.90,26.69,26.51,46.08$, 51.77 , and $45.37 \%$, respectively (Table 1 ).

The mean $\mathrm{F}_{\mathrm{LF}}$ value of $C$. dubliniensis isolates unexposed to antimycotics was 0.347 . Brief exposure to nystatin, amphotericin B, caspofungin, ketoconazole, fluconazole, and chlorhexidine gluconate significantly $(p<$ 0.05 to $p<0.001)$ increased the susceptibility of the isolates to lactoferrin, yielding mean $\mathrm{F}_{\mathrm{LF}}$ values of 0.394 , $0.404,0.408,0.415,0.421$, and 0.412 , respectively (Table 2). Thus, exposure to nystatin, amphotericin B, caspofungin, ketoconazole, fluconazole, and chlorhexidine gluconate provided mean percentage increase of susceptibility to lactoferrin by $13.54,16.43,17.58,19.60,21.32$, and $18.73 \%$, respectively (Table 2 ). The mean $\mathrm{F}_{\mathrm{LF}}$ value of C. albicans isolates unexposed to antimycotics was 0.357 . Brief exposure to nystatin, amphotericin B, caspofungin, ketoconazole, fluconazole, and chlorhexidine gluconate significantly $(p<0.05$ to $p<0.001)$ increased the susceptibility of the isolates to lactoferrin, yielding mean $\mathrm{F}_{\mathrm{LF}}$ values of $0.400,0.409,0.413,0.423,0.429$, and 0.420 , respectively (Table 2). Thus, exposure to nystatin, amphotericin $\mathrm{B}$, caspofungin, ketoconazole, fluconazole, and chlorhexidine gluconate provided mean percent increase in susceptibility to lactoferrin by $12.04,14.56,15.68,18.48$, 20.16 , and $17.65 \%$, respectively (Table 2 ).

\section{Discussion}

As the frequency of oral as well as systemic candidiasis-associated C. dubliniensis increases, there is a concomitant need for improvement in the pharmacodynamics of drugs with antifungal properties to manage such 
Table 1. The antifungal activity of lysozyme $\left(\mathrm{F}_{\mathrm{LZ}}\right)$ on oral Candida dubliniensis and Candida albicans isolates $(n=20$ each) following brief exposure to nystatin (NYS), amphotericin B (AMP-B), caspofungin (CASPO), ketoconazole (KETO), fluconazole (FLU), and chlorhexidine (CHX)

\begin{tabular}{|c|c|c|c|c|c|c|c|}
\hline Isolates & Control & NYS & AMP-B & CASPO & KETO & FLU & $\mathrm{CHX}$ \\
\hline \multicolumn{8}{|l|}{ C. dubliniensis } \\
\hline Mean & 0.550 & 0.602 & 0.714 & 0.710 & 0.821 & 0.848 & 0.820 \\
\hline SD & 0.029 & 0.020 & 0.021 & 0.019 & 0.028 & 0.037 & 0.030 \\
\hline Increase in sensitivity & & 9.45 & 30.82 & 30.04 & 50.64 & 55.60 & 50.18 \\
\hline$p$ value & & $<0.05$ & $<0.001$ & $<0.001$ & $<0.001$ & $<0.001$ & $<0.001$ \\
\hline SEM & 0.005 & 0.004 & 0.004 & 0.004 & 0.005 & 0.005 & 0.006 \\
\hline $\mathrm{SD}$ & 0.021 & 0.020 & 0.020 & 0.017 & 0.023 & 0.024 & 0.028 \\
\hline Increase in sensitivity & & 8.90 & 26.69 & 26.51 & 46.08 & 51.77 & 45.37 \\
\hline$p$ value & & $<0.05$ & $<0.001$ & $<0.001$ & $<0.001$ & $<0.001$ & $<0.001$ \\
\hline
\end{tabular}

Percent increase in sensitivity to lysozyme (\%) following exposure to these drugs is given at the bottom of the table. All values indicate the average of three experiments done in duplicate.

Table 2. The antifungal activity of lactoferrin $\left(\mathrm{F}_{\mathrm{LF}}\right)$ on oral Candida dubliniensis and Candida albicans isolates following brief exposure to nystatin (NYS), amphotericin B (AMP-B), caspofungin (CASPO), ketoconazole (KETO), fluconazole (FLU), and chlorhexidine (CHX)

\begin{tabular}{|c|c|c|c|c|c|c|c|}
\hline Isolates & Control & NYS & AMP-B & CASPO & KETO & FLU & $\mathrm{CHX}$ \\
\hline \multicolumn{8}{|l|}{ C. dubliniensis } \\
\hline Mean & 0.347 & 0.394 & 0.404 & 0.408 & 0.415 & 0.421 & 0.412 \\
\hline SEM & 0.012 & 0.011 & 0.012 & 0.012 & 0.012 & 0.012 & 0.012 \\
\hline $\mathrm{SD}$ & 0.053 & 0.050 & 0.052 & 0.053 & 0.055 & 0.054 & 0.054 \\
\hline Increase in sensitivity & & 13.54 & 16.43 & 17.58 & 19.60 & 21.32 & 18.73 \\
\hline$p$ value & & $<0.05$ & $<0.05$ & $<0.01$ & $<0.01$ & $<0.001$ & $<0.01$ \\
\hline \multicolumn{8}{|l|}{ C. albicans } \\
\hline Mean & 0.357 & 0.400 & 0.409 & 0.413 & 0.423 & 0.429 & 0.420 \\
\hline SEM & 0.009 & 0.008 & 0.008 & 0.010 & 0.010 & 0.010 & 0.007 \\
\hline $\mathrm{SD}$ & 0.041 & 0.036 & 0.035 & 0.045 & 0.044 & 0.043 & 0.031 \\
\hline Increase in sensitivity & & 12.04 & 14.56 & 15.68 & 18.48 & 20.16 & 17.65 \\
\hline$p$ value & & $<0.05$ & $<0.05$ & $<0.05$ & $<0.01$ & $<0.001$ & $<0.01$ \\
\hline
\end{tabular}

Percent increase in sensitivity to lactoferrin (\%) following exposure to these drugs is given at the bottom of the table. All values indicate the average of three experiments done in duplicate.

infections. In this study, brief exposure of C. dubliniensis oral isolates to a sub-MIC dose of the drugs was attempted, so as to mimic the oral conditions in vitro as done previously [18-24]. Thereafter, the changes in susceptibility to the antifungal effect of two salivary constituents, lysozyme and lactoferrin, were evaluated on this Candida species (in addition, as C. dubliniensis and C. albicans are phenotypically similar, C. albicans oral isolates were also exposed to the same conditions for the purpose of comparison of results between these two Candida species).
In our study, the MIC values of all tested antifungal agents were within the range of the reference strain, which implies that the Candida isolates tested were susceptible to the drugs used. Furthermore, these values were within the MIC range obtained in previous investigations of C. dubliniensis and C. albicans isolates [18-20,23].

In the present study, it was noted that $C$. dubliniensis and C. albicans isolates significantly exhibited increased susceptibility to both lysozyme and lactoferrin when preexposed to sub-MIC concentration of 6 different agents 
with antifungal properties for a brief period. In a previous study, too, though not significant for some of the tested isolates, an overall increase in susceptibility to both these salivary constituents were reported following brief exposure of C. albicans and C. tropicalis isolates to nystatin, amphotericin B, and fluconazole [24].

The precise mechanism by which lysozyme interrelates with the fungal cell wall is not clear. However, two distinctive complementary mechanisms have been suggested. Firstly, the enzymatic hydrolysis of N-glycosidic bonds that link polysaccharides and structural proteins of the candidal cell wall may result in consequent injury to the cytoplasmic membrane [27]. Secondly, impairment of the plasma lemma of the yeast cell may be followed by activation of mannan synthetase, chitin synthetase, and probably other enzymes that code for the yeast cell wall constituents [27]. In addition, the fungicidal effect of lactoferrin and apo-lactoferrin-mediated killing of Candida has been shown to depend on factors such as $\mathrm{pH}$, temperature, and incubation period [26]. Again, the accurate mode of action of lactoferrin on Candida is not clear although it is speculated that sequestration of elemental iron and its deprivation may play a role [24]. It has also been noted that lactoferrin interacts with the candidal cell surface causing extracellular leakage of proteins and the formation of surface blebs [24].

As the precise mechanism by which lysozyme and lactoferrin interact with the candidal cell wall is not yet clear, it is not possible to definitively explain the higher sensitivity of C. dubliniensis and C. albicans isolates to both lysozyme and lactoferrin pre-exposed to drugs with antifungal properties. However, we speculate that it may be partially related to the mechanism of action of these drugs on Candida contributing to a synergistic effect with these two salivary constituents. For instance, polyenes (nystatin and amphotericin B) bind to sterol components in the cell wall of Candida and make it more permeable $[16,17]$. Also, it has been advocated that the creation of sterols or their precursors may be repressed by the polyenes [16, 17]. Furthermore, studies have revealed internally misshapen cells with an intact cell wall leaving "ghost-like" cells and collapsed Candida cells following exposure to sub-fungicidal concentrations of nystatin $[16,17]$. In addition, microbial appendices such as the outer membrane protein, lipoprotein, phospholipid, lipopolysaccharide, and fimbriae may be altered by polyenes [16, 17]. Similarly, caspofungin affects the enzyme responsible for biosynthesis of $\beta-1,3$ glucan ensuing in disrupting the integrity of the yeast cell wall $[16,17,23]$ and azoles (ketoconazole and fluconazole) act by altering the fungal cell membranes blocking the $14 \alpha$-demethylation step in the biosynthesis of ergosterol. The consequential exhaustion of ergosterol and accretion of 14a-methyl sterols leads to alterations in a number of membrane-associated functions $[16,17]$. Scanning and transmission electron micrographic studies have exhibited that the antifungal effect of chlorhexidine is most likely due to the loss of cytoplasmic components and coagulation of nucleoproteins and associated morphological changes in the cell wall structure $[16,17]$. Hence, taken together, the increased susceptibility to both lysozyme and lactoferrin following exposure to polyenes, echinocandins, azoles, and chlorhexidine may be related to the pharmacodynamics of these drugs on the cell wall of Candida.

One previous report has described the influence of nystatin, amphotericin B, and fluconazole on the susceptibility of C. albicans and C. tropicalis isolates to lysozyme and lactoferrin [24], but the current study is first to evaluate the effect of lactoferrin and lysozyme on a large number of $C$. dubliniensis and C. albicans isolates $(n=40)$ obtained from a single geographic location, following exposure to drugs with a wide array of antifungal pharmacodynamics, ranging from polyenes, echinocandins, azoles, and chlorhexidine. However, we studied only two putative salivary antifungal defenses; therefore, these findings cannot be interpreted as fully expressive of the oral milieu because other nonspecific salivary defense factors such as histatins, cystatins, lactoperoxidase, chromogranin A as well as specific defenses such as secretary IgA are likely to operate in vivo [28]. However, our data indicate that exposure to drugs such as polyenes, echinocandins, azoles as well as chlorhexidine gluconate may enhance the susceptibility of Candida to lysozyme and lactoferrin, thereby increasing the defense capability of these salivary constituents towards this yeast. Interestingly, resistance of C. dubliniensis to fluconazole, voriconazole, and itraconazole has been documented previously [8-10]. Furthermore, resistance of C. dubliniensis to 5 -fluorocytosine in isolates obtained from Kuwait and the Middle East region has also been documented [11, 12]. Occurrence of such resistance indicates the need for possible alternative therapeutic modalities which may facilitate the management of candidiasis. In this context, the current study suggests that exposure to polyene, echinocandin, and azole antifungal agents and chlorhexidine may induce a synergistic effect by enhancing the susceptibility of C. dubliniensis to naturally occurring salivary constituents such as lysozyme and lactoferrin, which may provide additional information about pharmacodynamics of these drugs on C. dubliniensis isolates (as well as 
C. albicans isolates). In addition, in our study there was no significant difference in the impact of antifungal drugs on the susceptibility of the two Candida species towards lysozyme and lactoferrin. This is not surprising as these two Candida species are considered phenotypically almost identical Candida species. However, the overall lower susceptibility of $C$. albicans species to lysozyme and lactoferrin compared to C. dubliniensis species indicates that $C$. albicans species are more resilient to the impact of both these salivary constituents even following exposure to antifungal drugs. This further adds credence to the fact that $C$. albicans is more virulent and pervasive than $C$. dubliniensis.

\section{Conclusion}

Brief exposure to nystatin, amphotericin B, caspofungin, ketoconazole, fluconazole, and chlorhexidine enhances the antifungal effect of lysozyme and lactoferrin on both C. dubliniensis and C. albicans isolates in vitro. However, more investigations with sessile Candida isolates encased in a biofilm, as opposed to the planktonic isolates used in the current study, are warranted to further augment the current evidence.

\section{Acknowledgments}

The work was supported by Kuwait University Research Grant No. DB 01/16. The technical support from Ms. Rachel Chandy and other laboratory staff, Department of Microbiology, Faculty of Medicine, Kuwait University, Kuwait is appreciated and thankfully acknowledged. The valuable contribution of Dr. Prem Sharma, Faculty of Medicine, Kuwait University, Kuwait to the statistical analysis of data is also much appreciated.

\section{Disclosure Statement}

The authors declare no conflict of interest.

\section{References}

1 Brandt ME, Harrison LH, Pass M, et al: Candida dubliniensis fungemia: the first four cases in North America. Emerg Infect Dis 2000; 6:46-49.

2 Manfredi M, McCullough MJ, Al-Karaawi $\mathrm{ZM}$, et al: The isolation, identification and molecular analysis of Candida spp. isolated from the oral cavities of patients with diabetes mellitus. Oral Microbiol Immunol 2002;17: 181-185.

3 Peltroche-Llacsahuanga $\mathrm{H}$, Dohmen $\mathrm{H}$, Haase G: Recovery of Candida dubliniensis from sputum of cystic fibrosis patients. Mycoses 2002;45:15-18.

4 Garcia J, Soch K, Matthew E, et al: Endocarditis caused by Candida dubliniensis. Am J Med Sci 2013;346:237-239.

5 Wellinghausen N, Moericke A, Bundschuh S, et al: Multifocal osteomyelitis caused by Candida dubliniensis. J Med Microbiol 2009;58: 386-390.

6 Andrew NH, Ruberu RP, Gabb G: The first documented case of Candida dubliniensis leptomeningeal disease in an immunocompetent host. BMJ Case Rep 2011;2011.pii: bcr0620114384.

7 Oksi J, Finnilä T, Hohenthal U, et al: Candida dubliniensis spondylodiscitis in an immunocompetent patient. Case report and review of the literature. Med Mycol Case Rep 2013;3: 4-7.
8 Schorling SR, Kortinga HC, Froschb M, et al: The role of Candida dubliniensis in oral candidiasis in human immunodeficiency virusinfected individuals. Crit Rev Microbiol 2000: 26:59-68.

9 Fanci R: Breakthrough Candida dubliniensis fungemia in an acute myeloid leukemia patient during voriconazole therapy successfully treated with caspofungin. J Chemother 2009;21:105-107.

10 Fleischhacker M, Pasligh J, Moran G, et al: Longitudinal genotyping of Candida dubliniensis isolates reveals strain maintenance, microevolution, and the emergence of itraconazole resistance. J Clin Microbiol 2010;48: 1643-1650.

11 Ahmad S, Khan ZU, Joseph L, et al: Genotypic heterogeneity and molecular basis of 5-flucytosine resistance among Candida dubliniensis isolates recovered from clinical specimens in Kuwait. Med Mycol 2012;50: 244-251.

$12 \mathrm{Al}$ Mosaid A, Sullivan DJ, Polacheck I, et al: Noval 5-flucytosine clade of Candida dubliniensis from Saudi Arabia and Egypt by Cd25 fingerprinting. J Clinic Microbiol 2005;43: 4026-4036.
13 Mehra T, Köberle M, Braunsdorf C, et al: Alternative approaches to antifungal therapies. Exp Dermatol 2012:21:778-782.

14 Nikawa H, Hamada T: Binding of salivary or serum proteins to Candida albicans in vitro. Arch Oral Biol 1990;35:571-573.

15 Samaranayake YH, Samaranayake LP, Pow $\mathrm{EH}$, et al: Antifungal effects of lysozyme and lactoferrin against genetically similar, sequential Candida albicans isolates from a human immunodeficiency virus-infected southern Chinese cohort. J Clin Microbiol 2001;39: 3296-3302.

16 Ellepola AN, Samaranayake LP: Antimycotic agents in oral candidosis: an overview: treatment of oral candidosis. Dent Update 2000; $27: 165-174$.

17 Ellepola AN, Samaranayake LP: Oral candidal infections and antimycotics. Crit Rev Oral Biol Med 2000;11:172-198.

18 Ellepola AN, Joseph BK, Altarakemah Y, et al: In vitro adhesion of oral Candida dubliniensis isolates to acrylic denture surfaces following brief exposure to sub-cidal concentrations of polyenes, azoles and chlorhexidine. Med Princ Pract 2015;24:58-64.

19 Ellepola AN, Chandy R, Khan ZU: Post-antifungal effect and adhesion to buccal epithelial cells of oral Candida dubliniensis isolates subsequent to limited exposure to amphotericin $\mathrm{B}$, ketoconazole and fluconazole. J Investig Clin Dent 2015;6:186-192. 
20 Ellepola AN, Joseph BK, Khan ZU: Changes in germ tube formation and cell surface hydrophobicity of oral Candida dubliniensis isolates following brief exposure to sub-cidal concentrations of polyene and azole antifungal agents. Mycoses 2013;56:463-470.

21 Ellepola AN, Khajah R, Jayatilake S, et al: Impact of brief exposure to antifungal agents on the post-antifungal effect and hemolysin activity of oral Candida albicans. J Appl Oral Sci 2015;23:412-418.

22 Ellepola AN, Samaranayake LP, Khan ZU: Extracellular phospholipase production of oral Candida albicans isolates from smokers, diabetics, asthmatics, denture wearers and healthy individuals following brief exposure to polyene, echinocandin and azole antimycotics. Braz J Microbiol 2016;47:911-916.

23 Ellepola AN, Chandy R, Khan ZU, et al: Caspofungin-induced in-vitro post-antifungal effect and its impact on adhesion related traits of oral Candida dubliniensis and Candida albicans isolates. Microbiol Immunol 2016;60: 160-167.

24 Anil S, Samaranayake LP: Impact of lysozyme and lactoferrin on oral Candida isolates exposed to polyene antimycotics and fluconazole. Oral Dis 2002;8:199-206.

25 Ellepola AN, Khan ZU, Joseph BJ, et al: Prevalence of Candida dubliniensis among oral
Candida isolates in patients attending the $\mathrm{Ku}$ wait University Dental Clinic. Med Princ Pract 2011;20:271-276.

26 Soukka T, Tenovuo J, Lenander-Lumikari M: Fungicidal effect of human lactoferrin against Candida albicans. FEMS Microbiol Lett 1992; 69:223-228.

27 Marquis G, Garzon S, Strykowski H, et al: Cell walls of normal and lysozyme-damaged blastoconidia of Candida albicans: localization of surface factor 4 antigen and vicinal-glycol staining. Infect Immun 1991;59:1312-1318.

28 Nieuw Amerongen AV, Veerman EC: Saliva the defender of the oral cavity. Oral Dis 2002; 8:12-22. 